\title{
Identification of resonant earthquake ground motion
}

\author{
ABBAS MOUSTAFA \\ Department of Civil Engineering, Minia University, Minia 61111, Egypt \\ e-mail: abbas.moustafa@yahoo.com
}

MS received 5 October 2009; revised 17 January 2010; accepted 22 March 2010

\begin{abstract}
Resonant ground motion has been observed in earthquake records measured at several parts of the world. This class of ground motion is characterized by its energy being contained in a narrow frequency band. This paper develops measures to quantify the frequency content of the ground motion using the entropy principle and the dispersion index. These measures are based on the geometric properties of the power spectral density function of the ground acceleration. The application of these measures to quantify the frequency content of random earthquake models is demonstrated first. Subsequently, these measures are used to quantify the frequency content of the ground acceleration for near-field records measured at rock and soil sites, short-duration and long-duration earthquakes.
\end{abstract}

Keywords. Disorder; dispersion index; entropy rate; frequency content; resonant ground motion, uncertainty.

\section{Introduction}

Earthquake ground motion is highly uncertain and difficult to be predicted (Geller et al 1997). Earthquake uncertainties include time, location, magnitude, intensity, and duration. These uncertainties can be divided into two main categories, referred to as aleatory and epistemic uncertainties (Wen et al 2003; Sigbjornsson \& Ambraseys 2004; Bommer \& Scherbaum 2005). Epistemic uncertainties result from the lack of knowledge to accurately describe the phenomenon. Obtaining new data and refining the modelling can reduce these uncertainties. Aleatory uncertainties are related to the inherent unpredictability of earthquake process which cannot be reduced. Uncertainty quantification of ground motion is necessary in reliability analysis and performance and optimal design of structures (Sasani \& Bertero 2000; Wen \& Song 2003). The increasing availability of recorded ground motions at many parts of the world and the ease in accessing this data from the Internet or in the form of CD-Rom pave the way for better understanding of earthquake ground motions. Table 1 lists some sources for strong ground motion records (Bozorgnia \& Campbel 2004).

A few studies have attempted to identify resonant or critical ground accelerations. For instance, the effective peak velocity of the ground motion has been used to define resonant records (Makris \& Black 2004; Amiri \& Dana 2005). Takewaki (2001) and Zhai \& Xie (2007) identified critical ground motion for nonlinear structures using inelastic time-history analysis. The effect of energy concentration of earthquake accelerations on the nonlinear response 


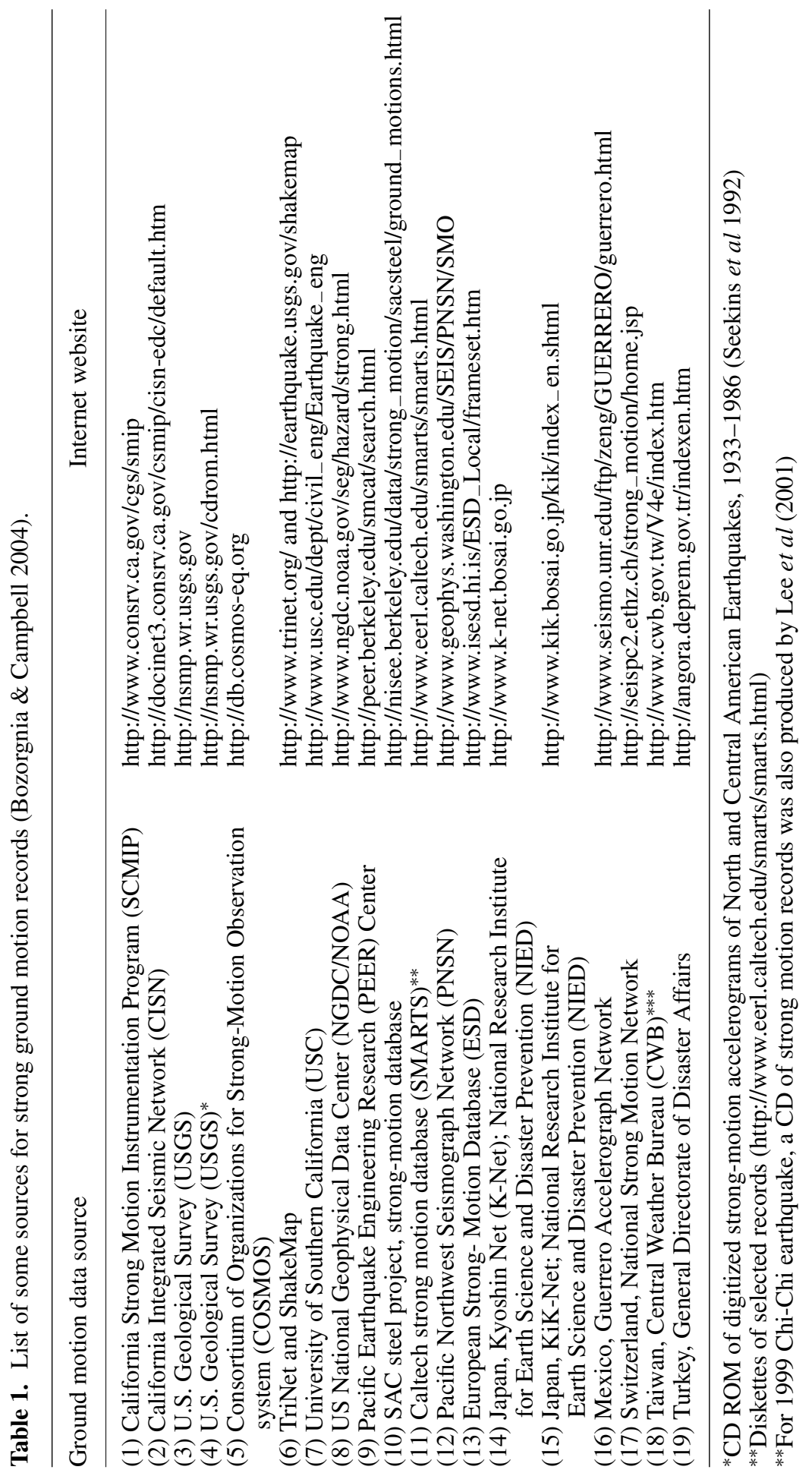


of RC structures has been recently studied (Cao \& Friswell 2009; Moustafa 2009). This paper develops quantitative measures to quantify the uncertainty or the amount of disorder in earthquake ground motions using the entropy principle and the dispersion index. These measures are based on the geometric properties of the power spectral density function (PSDF) of the ground acceleration, and are used for quantifying the frequency content of the ground motion.

The idea of using the entropy to measure the amount of information in random signals sent along a transmission line was proposed by Shannon (1948). The literature on the use of entropy in engineering is vast (Kapur 1993; Papoulis \& Pillai 2002). The entropy principle is often associated with uncertainty or incomplete information. In the context of earthquake engineering, Manohar \& Sarkar (1995) recognized the importance of entropy in modelling critical earthquake loads. They derived the critical random earthquake by maximizing the structure's response and the entropy at the same time. This author placed explicit constraints on the entropy of the PSDF of the critical ground motion models (Abbas \& Manohar 2002; 2007). This constraint was quantified from past recorded accelerograms and was shown to produce realistic ground motion. Readers can refer Iyengar \& Manohar (1987); Iyengar (1989); Manohar \& Sarkar 1995; Abbas \& Manohar 2002 for more details on the critical excitation method. The next section demonstrates the quantification of uncertainty for stationary random models of ground accelerations.

\section{Quantification of uncertainty in probabilistic earthquake models using entropy}

Entropy in its basic form is a measure of uncertainty rather than a measure of information. For instance, the entropy of a random variable $x$ is a measure of the uncertainty associated with that random variable. Thus, the uncertainty in a dice experiment is larger than that in a coin experiment. When entropy of a random variable is large, the uncertainty as to the value of the random variable is also large. An example is a normal random variable with large coefficient of variation. On the other hand, the entropy or uncertainty of a deterministic quantity is zero. The same concept applies to random processes. The entropy rate or the amount of disorder of a stationary Gaussian random process $\ddot{u}_{g}(t)$ is given as (Papoulis \& Pillai 2002):

$$
H=\ln \sqrt{2 \pi e}+\frac{1}{2\left(\omega_{u}-\omega_{0}\right)} \int_{\omega_{0}}^{\omega_{u}} \ln S(\omega) d \omega,
$$

where $\left(\omega_{0}, \omega_{u}\right), S(\omega)$ are the frequency range and the PSDF of the ground acceleration, $e$ is a constant $(e=2.72)$ and the term $\ln \sqrt{2 \pi e}$ represents a reference level from which entropy is measured. Equation (1) reveals that the entropy rate of a stationary Gaussian process is constant. Thus, when the entropy of $\ddot{u}_{g}(t)$ is large, the uncertainty as to the value of $\ddot{u}_{g}(t)$ at discrete time instants is also large. Conversely, the uncertainty of a pulse-like signal of random amplitude and a single frequency (e.g. $\ddot{u}_{g}(t)=A \sin (0 \cdot 50 \pi t)$ ) is almost zero. In other words, the energy of resonant or pulse-like time series of a single or a few frequencies is well ordered in the frequency domain, while that of a time signal composed of several frequencies will be disordered. Note that two stationary random processes with the same energy (i.e. same variance) need not have the same entropy. This is because, entropy depends on the distribution of the spectral amplitude of the PSDF at different frequencies (see equation 1). Hence, entropy can reflect the frequency range of the ground acceleration (Abbas \& Manohar 2002; 2007).

In this context, it may be emphasized that the time history and the Fourier amplitude spectra of the ground acceleration may reflect the frequency content of the ground motion 
qualitatively. The entropy rate and the dispersion index introduced in this paper, as will be shown in the next sections, provide quantitative measures for the frequency content of the ground motion. Furthermore, the time history or the Fourier amplitude spectra of the ground acceleration does not provide an estimate for comparing the frequency content of a set of acceleration records. On the other hand, the proposed measures can be effectively used to rank the records based on their frequency contents. This aspect is of relevance in the selection of earthquake records for time-history analysis of structures (Moustafa et al 2010).

To gain more insights into the concept of entropy in quantifying uncertainty of ground motions we derive the entropy rate for probabilistic models of stationary Gaussian ground motions below.

\subsection{Band-limited white noise model}

The band-limited white noise process has been used as an approximation for earthquake and wind loads. This process has constant spectral amplitude in the specified frequency range. Considering a band-limited stationary random process of intensity $s_{0}$ in the frequency range $\left(\omega_{0}, \omega_{u}\right)$, the entropy of this process is given as (see equation 1$)$ :

$$
\begin{aligned}
H & =\ln \sqrt{2 \pi e}+\frac{1}{2\left(\omega_{u}-\omega_{0}\right)} \int_{\omega_{0}}^{\omega_{u}} \ln s_{0} d \omega \\
& =\ln \sqrt{2 \pi e}+\frac{1}{2\left(\omega_{u}-\omega_{0}\right)} \times\left(\omega_{u}-\omega_{0}\right) \ln s_{0}=\ln \sqrt{2 \pi e}+\frac{\ln s_{0}}{2} .
\end{aligned}
$$

Therefore, the spectral parameter $s_{0}$ defines the entropy or the amount of disorder for the band-limited process.

\subsection{Exponential PSD model}

Consider a stationary model of earthquake acceleration defined in terms of the auto-correlation function and the associated PSDF as (Lin 1967):

$$
R(\tau)=s_{0} \exp \left(-a \tau^{2}\right) ; S(\omega)=\frac{s_{0}}{2 \sqrt{\pi a}} \exp \left(\frac{-\omega^{2}}{2 a}\right),
$$

where $a$ is a constant and $\tau$ is the time-lag. Accordingly, equation (1) leads to:

$$
H=\ln \sqrt{2 \pi e}+\frac{1}{2\left(\omega_{u}-\omega_{0}\right)} \int_{\omega_{0}}^{\omega_{u}} \ln \left[\frac{s_{0}}{2 \sqrt{\pi a}} \exp \left(\frac{-\omega^{2}}{2 a}\right)\right] d \omega .
$$

Carrying out the integration and performing necessary reductions, we get:

$$
H=\ln \sqrt{2 \pi e}+\frac{1}{2}\left[\ln \left(\frac{s_{0}}{2 \sqrt{a \pi}}\right)-\frac{1}{6 a}\left(\omega_{u}^{2}+\omega_{u} \omega_{0}+\omega_{0}^{2}\right)\right] .
$$

Thus, the parameters $a, s_{0}, \omega_{0}, \omega_{u}$ define the entropy of this random process.

\subsection{Kanai-Tajimi model}

The Kanai-Tajimi model has been widely used in modelling earthquake ground motions due to its physical meaning and simplicity. The PSDF of the ground acceleration is obtained by 
passing a band-limited white noise through a filter that represents the soil layer above the rock level, given as (Kanai 1957; Tajimi 1960):

$$
S(\omega)=s_{0} \frac{\omega_{g}^{4}+4 \eta_{g}^{2} \omega_{g}^{2} \omega^{2}}{\left(\omega_{g}^{2}-\omega^{2}\right)^{2}+4 \eta_{g}^{2} \omega_{g}^{2} \omega^{2}},
$$

where, $s_{0}, \eta_{g}, \omega_{g}$ are the intensity of the PSDF at the rock level and the damping and natural frequency of the soil layer, respectively. The entropy rate of the Kanai-Tajimi model can be estimated numerically instead of carrying out the integration of equation (1) analytically since it leads to a lengthy expression.

This section demonstrated the quantification of the entropy rate as a measure of uncertainty of probabilistic stationary ground motion models. It is shown that the entropy rate of a stationary random process is constant. Note that the entropy of a non-stationary random process is a time-dependent function. The next subsection explains the quantification of relative entropy of two random processes which is used later in identifying resonant accelerograms.

\subsection{Relative entropy rate}

To compare uncertainty or amount of disorder of different earthquake acceleration models, we measure the entropy rate of the stationary process $\ddot{u}_{g}(t)$ with reference to a band-limited white noise process $\ddot{z}_{g}(t)$ of spectral intensity $s_{0}$. This is known as the relative entropy rate of the two random processes $\ddot{u}_{g}(t)$ and $\ddot{z}_{g}(t)$. Thus, assuming that $\ddot{u}_{g}(t)$ is independent of $\ddot{z}_{g}(t)$, the increase in the entropy rate when $\ddot{u}_{g}(t)$ is added to $\ddot{z}_{g}(t)$ is given as (Abbas \& Manohar 2002; 2007):

$$
\Delta H=\frac{1}{2\left(\omega_{u}-\omega_{0}\right)} \int_{\omega_{0}}^{\omega_{u}} \ln \left[1+\frac{S(\omega)}{s_{0}}\right] d \omega .
$$

Hence, equation (7) can be used to estimate the entropy rate $\Delta H$ of the random process $\ddot{u}_{g}(t)$ measured from a band-limited random process $\ddot{z}_{g}(t)$ as a reference level.

We now calculate the entropy rate for the narrow-band, the Kanai-Tajimi and the bandlimited models, described in the previous sub-sections, from a reference band-limited random process of intensity $0.02 \mathrm{~m}^{2} / \mathrm{s}^{3}$. The PSDF for each of these three models is normalized such that it possesses unit area (see figure 1). This normalization implies equality of the energy
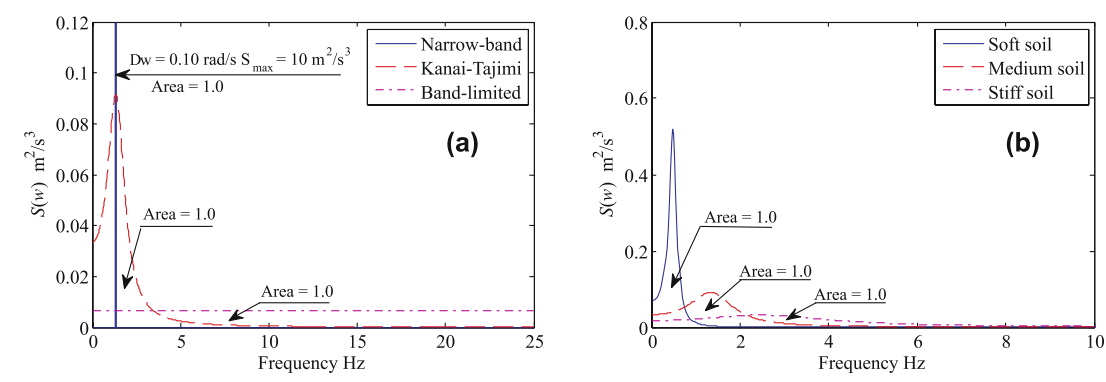

Figure 1. (a) Power spectral density function for alternative earthquake models; (b) Kanai-Tajimi power spectral density function for different soil types. 
Table 2. Entropy and dispersion indices for alternative earthquake models and different soil site conditions.

\begin{tabular}{|c|c|c|c|c|c|c|}
\hline \multirow[b]{3}{*}{ Earthquake model } & \multicolumn{6}{|c|}{ Soil type } \\
\hline & \multicolumn{2}{|c|}{ Soft soil } & \multicolumn{2}{|c|}{ Medium soil } & \multicolumn{2}{|c|}{ Stiff soil } \\
\hline & Entropy & Dispersion & Entropy & Dispersion & Entropy & Dispersion \\
\hline Narrow-band* & 0.0319 & 0.0785 & 0.0319 & 0.0785 & 0.0319 & 0.0785 \\
\hline Kanai-Tajimi** & $0 \cdot 2600$ & 4.0869 & 0.5353 & $14 \cdot 7227$ & 0.7199 & $26 \cdot 2513$ \\
\hline Band-limited & 0.8673 & $45 \cdot 2997$ & 0.8673 & $45 \cdot 2997$ & $0 \cdot 8673$ & $45 \cdot 2997$ \\
\hline 1940 Elecntro & - & - & 0.5112 & 8.6923 & - & - \\
\hline
\end{tabular}

* $\omega_{g}=\pi, 3 \pi, 6 \pi$ for soft, medium and stiff soil, respectively

** $\omega_{g}=\pi, 3 \pi, 6 \pi$ and $\eta_{g}=\pi, 3 \pi, 6 \pi$ for soft, medium and stiff soil, respectively

from the three models (Arias 1970). The parameters of the Kanai-Tajimi model $\omega_{g}, \eta_{g}$ are taken as $\pi \mathrm{rad} / \mathrm{s}, 0.20$ for soft soil, $3 \pi \mathrm{rad} / \mathrm{s}, 0.40$ for medium soil and $6 \pi \mathrm{rad} / \mathrm{s}, 0.60$ for stiff soil, see table 2 (Der Kiureghian 1996). Additionally, the spectral intensity at the rock level is taken as $s_{0}=0.02 \mathrm{~m}^{2} / \mathrm{s}^{3}$ and the central frequency of the narrow-band process is taken as $\omega_{g}=\pi, 3 \pi, 6 \pi \mathrm{rad} / \mathrm{s}$ for soft, medium and stiff soil, respectively. The numerical results are shown in table 2 . Based on a careful investigation of these results, the following observations are made:

- The narrow-band signal possesses the smallest entropy rate, and, thus the associated amount of disorder is the lowest among the three models. In other words, the PSDF of this model is well ordered in the frequency range $\left(\omega_{0}, \omega_{u}\right)$ since the energy of the signal is concentrated at a single frequency. This process represents a pulse-like or resonant signal (Moustafa 2010). Note also that the central frequency of the narrow-band signal has no effect on the value of the entropy rate (see table 2). Thus, the entropy rate of narrow-band processes with equal intensity is invariant regardless of the resonant frequency.

- The band-limited signal possesses the highest entropy rate among the three models. Thus, the associated amount of disorder is large. This is because the energy of the process is well distributed across the frequency range $\left(\omega_{0}, \omega_{u}\right)$. This scenario represents a random process composed of several harmonic signals with different frequencies (e.g. $\left.\ddot{u}_{g}(t)=\sum_{i=1}^{N} A_{i} \cos \left(\omega_{i} t+\phi_{i}\right)\right)$. Thus, $\ddot{u}_{g}(t)$ possesses a large amount of disorder since the energy is well represented at all frequencies.

- The Kanai-Tajimi acceleration is significantly disordered. This result is consistent since the PSDF is reasonably distributed across a significant frequency range (see figure 1a). Additionally, the entropy for the case of soft soil is small while that for stiff soil is large. This is expected since the PSDF for soft soil is narrow-banded compared to that for stiff soil that is distributed across a wider frequency range (see figure $1 \mathrm{~b}$ ).

- The entropy rate for the Kanai-Tajimi model is bounded between the entropy rate of the narrow-band white noise and that for the band-limited noise for all soil types (see table 2). This result is important since it provides lower and upper bounds on the amount of disorder for the Kanai-Tajimi acceleration model. These bounds can be used in validating the mathematical models of earthquake ground motions. 
In general, real earthquake accelerations have energies distributed across a significant wide frequency range. This is because the earthquake energy released at the source gets amplified and filtered due to path and local soil effects. Such accelerograms possess significant amount of disorder or uncertainty. However, some real accelerograms show the resonance nature, and thus the associated entropy rate is small. An example of this scenario is near-fault earthquakes with directivity effects (Housner \& Hudson 1958; Anderson \& Bertero 1987; Sasani and Bertero 2000; Kalkan \& Kunnath 2008, Moustafa 2010). This is because, the ground acceleration measured near the source is influenced, mainly, by the source mechanism.

This section explained the quantification of the uncertainty or the amount of disorder for probabilistic earthquake acceleration models. It is shown that the narrow-band white noise and the band-limited white noise processes provide lower and upper bounds on the entropy rate of the Kanai-Tajimi acceleration model. The entropy rate of recorded accelerations is estimated below.

\subsection{Quantification of uncertainty in recorded accelerograms using entropy principle}

This section demonstrates the quantification of the entropy index of recorded ground motions. Consider a recorded earthquake acceleration $\ddot{x}_{g}(t)$ that is represented as:

$$
\ddot{x}_{g}(t)=e(t) \ddot{u}_{g}(t)=A_{0}\left[\exp \left(-\alpha_{1} t\right)-\exp \left(-\alpha_{2} t\right)\right] \ddot{u}_{g}(t) .
$$

Here $\ddot{u}_{g}(t)$ represents a steady-state function and $e(t)$ is an envelope function that controls the non-stationarity of $\ddot{x}_{g}(t)$. The envelope parameters $A_{0}, \alpha_{1}, \alpha_{2}$ can be estimated by matching the transient trend of the earthquake acceleration. Subsequently, the stationary part of the ground acceleration $\ddot{u}_{g}(t)$ is obtained by dividing $\ddot{x}_{g}(t)$ by the estimated envelope. The PSDF of $\ddot{u}_{g}(t)$ is then determined. This is followed by the estimation of the entropy rate using equation (7).

Figure 2 shows the PSDF for four ground accelerations. The first acceleration represents a sample narrow-band signal $\ddot{x}_{g}(t)=e(t) A \cos \omega_{g} t$, with random amplitude $A$ and central frequency $\omega_{g}=3 \pi$. The second acceleration represents a sample band-limited model $\ddot{x}_{g}(t)=$ $e(t) \sum_{i=1}^{N} A_{i} \cos \left(\omega_{i} t+\phi_{i}\right)$. The third signal is a simulated acceleration from the KanaiTajimi model of equation (6) $\left(\omega_{g}=3 \pi \mathrm{rad} / \mathrm{s} ; \eta_{g}=0.40\right)$. The fourth acceleration represents the popular $1940 \mathrm{El}$ Centro NS accelerogram that is usually adopted by engineers for time history analysis of structures (PEER 2005). The stationary components $\ddot{u}_{g}(t)$ for the first three models are modulated by the envelope function $e(t)$ that matches the transient trend of El Centro record. All accelerations are normalized to unit intensity $\int_{0}^{\infty}\left[\ddot{x}_{g}(t)\right]^{2} d t=1 \cdot 0$ (Arias 1970).

The relative entropy of each of these accelerations, from a band-limited acceleration of spectral intensity $0.02 \mathrm{~m}^{2} / \mathrm{s}^{3}$, is determined. The numerical values are found to be 0.03 for the narrow-band acceleration, 0.87 for the band-limited acceleration, 0.54 for the simulated Kanai-Tajimi acceleration and 0.51 for the El Centro record (see table 2). These results confirm that the amount of disorder from the Kanai-Tajimi model is bounded between the entropy of the narrow-band acceleration and the entropy of the band-limited acceleration. Interestingly, the amount of disorder of the actual ground acceleration is also bounded by the narrow-band and the band-limited signals. Note that the narrow-band signal represents resonant or pulse-like acceleration that is poor in frequency content. The band-limited signal, on the other hand, represents an acceleration that is rich in the frequency content. The next section explains the quantification of the dispersion index of recorded ground accelerations. 

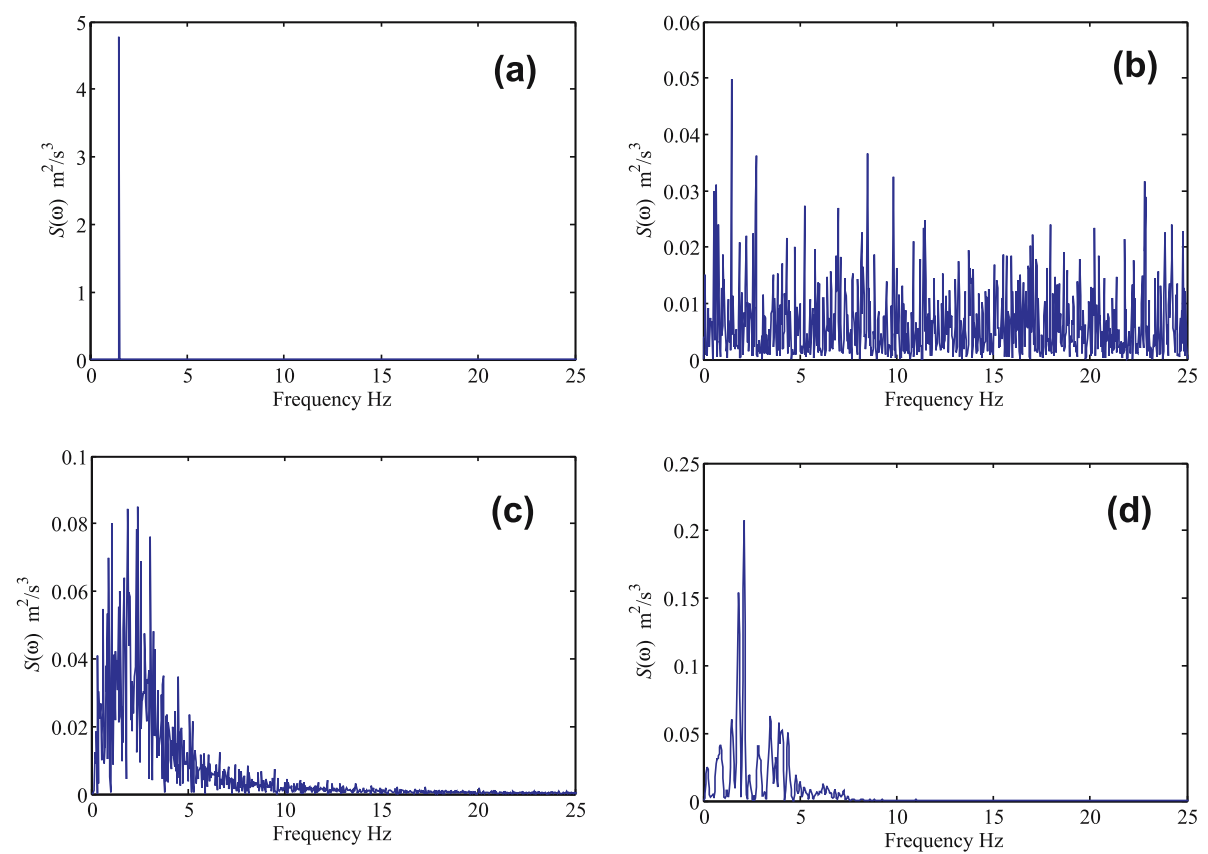

Figure 2. Power spectral density function for ground acceleration (a) narrow-band, (b) band-limited, (c) Kanai-Tajimi, (d) 1940 El Centro NS.

\section{Quantification of uncertainty in ground motion using dispersion index}

Consider a zero mean stationary Gaussian random process $\ddot{u}_{g}(t)$ defined in terms of the onesided PSDF $S(\omega)$ (see figure 3). The $i$ th frequency is defined as:

$$
\omega_{i}=\left(\frac{\int_{0}^{\infty} S(\omega) d \omega}{\int_{0}^{\infty} \omega^{i} S(\omega) d \omega}\right)^{i} ; i=1,2, \ldots, N .
$$

Here $\omega_{1}=\omega_{c}$ is the central or dominant frequency of the ground acceleration that reflects the influence of the soil condition. $\omega_{2}$ indicates where the spectral mass of the PSDF is located along the frequency range. The frequency $\omega_{s}$ can be defined as the radius of gyration of $S(\omega)$ about the frequency origin $\omega_{c}$, given by (Vanmarcke 1972; 1976):

$$
\omega_{s}=\sqrt{\omega_{2}^{2}-\omega_{1}^{2}}
$$

The radius of gyration $\omega_{s}$ is a measure of dispersion of the PSDF about the central frequency (Vanmarcke 1972; 1976). The application of equation (10) to earthquake records requires the determination of the PSDF of the stationary acceleration which has been demonstrated in the previous section. We estimate the dispersion index for the four acceleration models discussed in the previous section (table 2 and figure 2). The numerical results listed in table 2 reveal that the dispersion index for the narrow-band and the band-limited signals provide lower and upper bounds on the dispersion index of the Kanai-Tajimi model and the El Centro record. These results agree with the results obtained on the entropy rate in the previous section. The next section provides practical applications of these measures in identifying resonant accelerograms. 


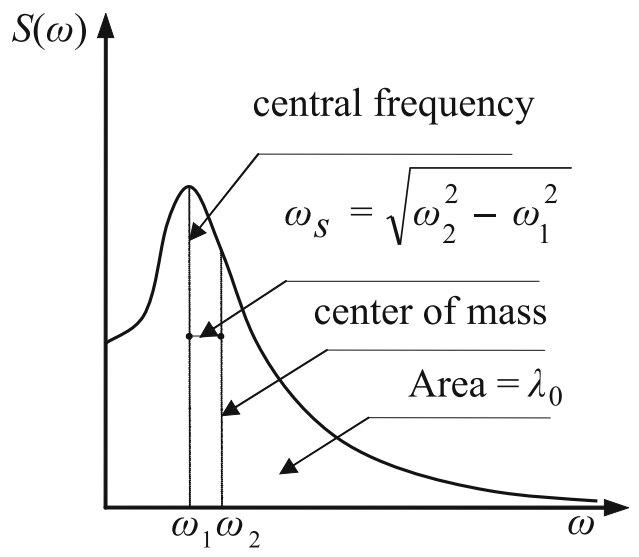

Figure 3. Power spectral density function for the stationary acceleration $\ddot{u}_{g}(t)$.

\section{Applications and numerical results}

To examine the applicability of using the entropy rate and the dispersion index in identifying resonant accelerograms we consider the four earthquake groups shown in table 3 . These records include near-fault accelerograms (site-source distance less than about $40 \mathrm{~km}$ ) measured at rock site (shear velocity $>750 \mathrm{~m} / \mathrm{s}$ ), near-fault accelerograms measured at soil site (shear velocity $<750 \mathrm{~m} / \mathrm{s}$ ), short-duration and long-duration earthquakes (PEER 2005). Note that the short- and the long-duration records have different soil conditions. Note also that the classification of these earthquake records has been carried out by Abrahamson et al (1998). The selection of records for different soil conditions and for different earthquake durations is meant to examine the robustness of the proposed measures to different earthquake characteristics. Ground accelerations of a minimum of $0.05 \mathrm{~g}$ peak ground acceleration are only considered. The two horizontal accelerations are considered in the numerical analyses. Table 3 provides information on magnitude, epicentral distance, PGA, Arias intensity, total duration and recording station for each of these records. Further details on these records can be found in Abrahamson et al (1998). In the numerical calculations, all accelerations are scaled to the same Arias intensity (Arias 1970).

The numerical results on entropy rate and dispersion index for each of these records are provided in table 3. Figures 4-7 show the PSDF for the stationary accelerations for the first four earthquakes from each group. The results reveal clearly that the entropy and the dispersion indices correlate well with each other and that they both successfully identify resonant records. It is seen that the narrow-band records possess the smallest entropy and dispersion indices (e.g. 1995 Kobe at OSAJ and 1994 Northridge at Sylmar). The ground accelerations that are rich in frequency content (e.g. 1992 Landers and 1986 N. Palm spring earthquakes) possess the largest entropy and dispersion. It is also evident that the two acceleration components of the same earthquake have different entropy and dispersion indices. This observation is consistent with the findings of Anderson \& Bertero (1987). This can be seen in the PSDF for the 1992 Landers earthquake which has a larger epicentral distance compared to other nearfault records measured at rock site (see figure 4). The short-duration earthquakes are seen to possess higher entropy and dispersion indices compared to the long-duration earthquakes. It is also remarkable that near-fault earthquakes measured at soil and rock sites have similar entropy and dispersion indices (see figures 4 and 5). In this context, it may be recalled that very close to the fault, the seismic source dominates the ground motion rather than site condition. 


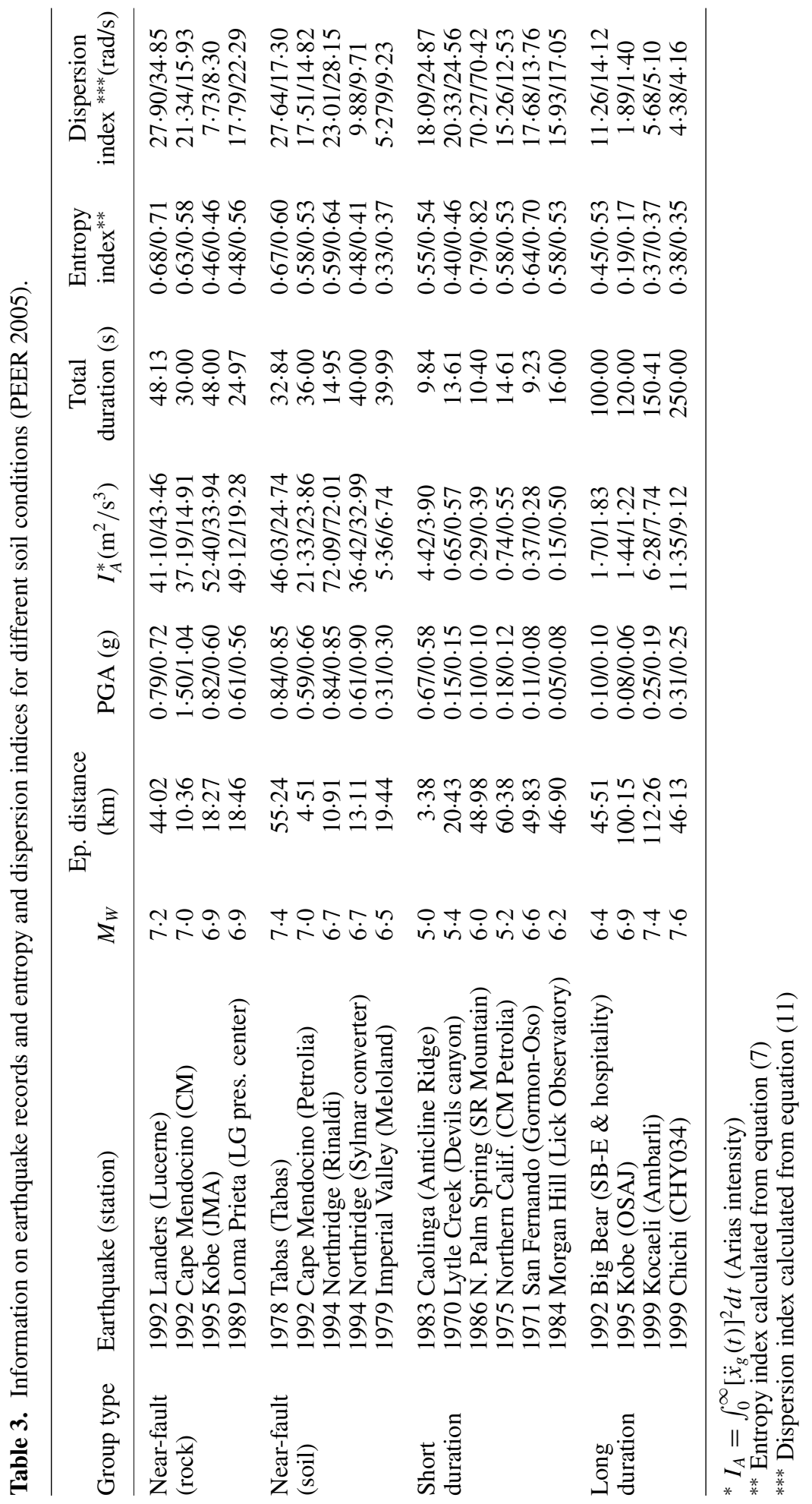



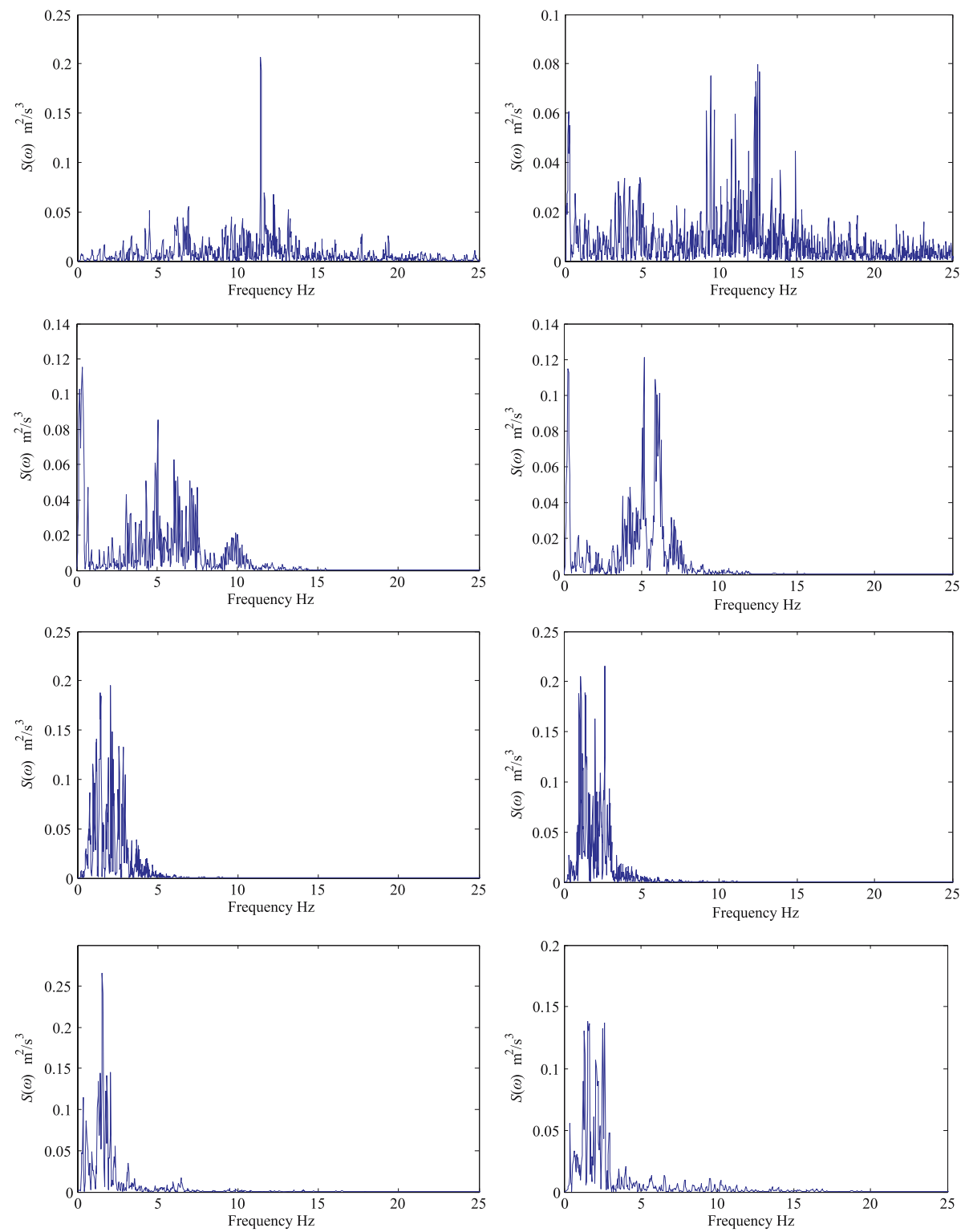

Figure 4. Power spectral density function: Near-fault (rock site).

In other words, the frequency content and duration of the earthquake records depend on the site-source distance and the source properties.

It may be noted that a limited number of records has been studied in this paper. However, for this limited number of records, the entropy rate and the dispersion index successfully identify the resonant records (i.e. the records with narrow frequency content), such as, the 1979 Imperial Valley and the 1995 Kobe earthquakes. Accordingly, these measures provide useful quantitative measures of the frequency content of the ground motion. 

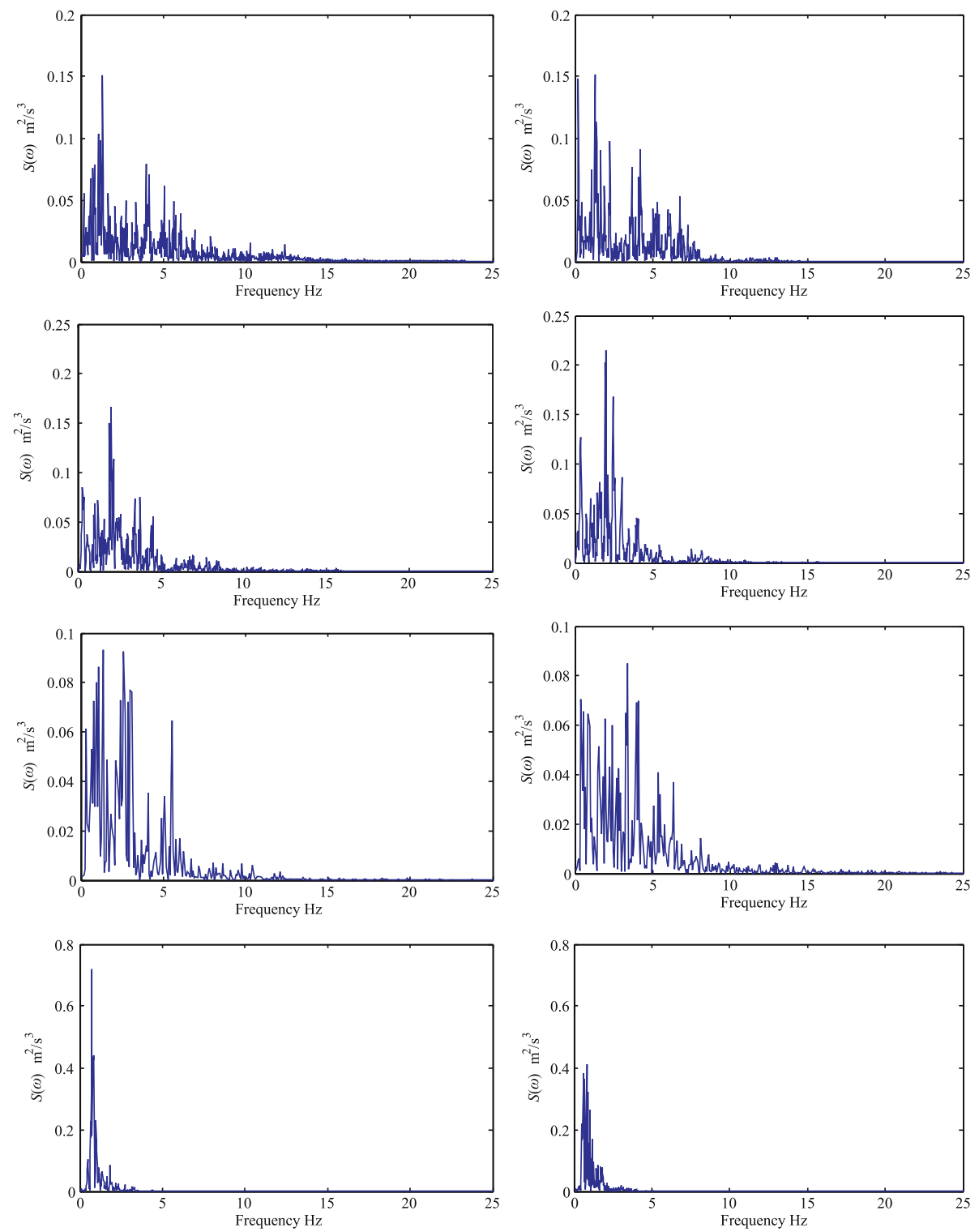

Figure 5. Power spectral density function: Near-fault (soil site).

To examine the effect of the frequency bandwidth of the ground acceleration on the structural damage, we consider the response analysis of three SDOF building structures of fundamental natural frequencies of $0.13,0.21$, and $0.41 \mathrm{~Hz}$. The yield displacement and strength are taken as $u_{y}=0.10 \mathrm{~m}, f_{y}=10^{4} \mathrm{~N}$ for the three structures and a viscous damping of 0.05 damping ratio is adopted. The material nonlinearity is modelled using elastic-plastic forcedeformation relation. Nonlinear dynamic analysis is carried out for each structure subjected to a single acceleration. All records are normalized to the same Arias intensity of $6.00 \mathrm{~m}^{2} / \mathrm{s}^{3}$. The Park and Ang damage index for each of these structures driven by the ground acceleration 

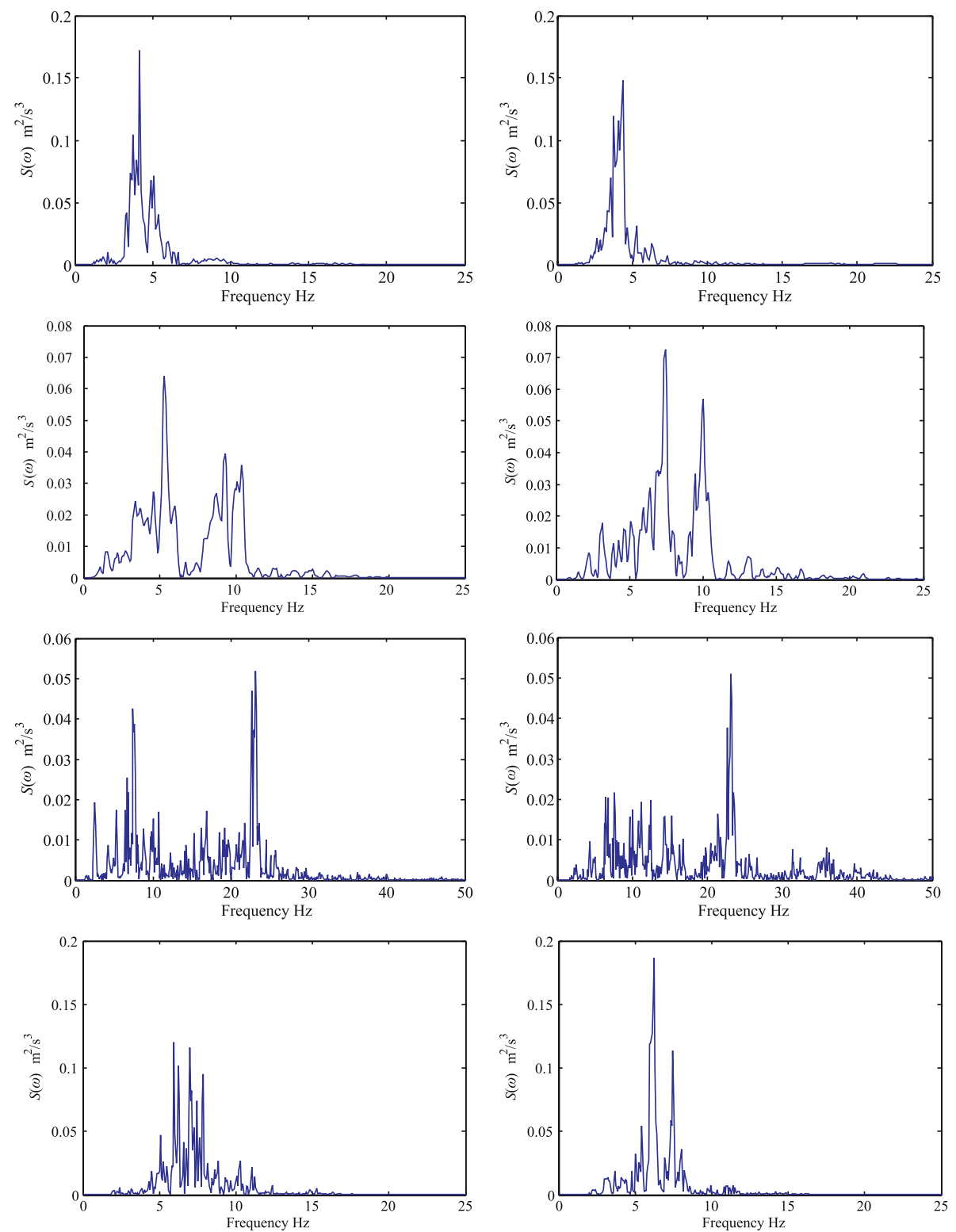

Figure 6. Power spectral density function: Short-duration records.

is calculated as follows (Park \& Ang 1985, Park et al 1985):

$$
D I_{\mathrm{PA}}=\frac{u_{\mathrm{max}}}{u_{u}}+\beta \frac{E_{\mathrm{H}}}{f_{y} u_{u}}=\frac{\mu_{\mathrm{max}}}{\mu_{u}}+\beta \frac{E_{\mathrm{H}}}{f_{y} u_{y} \mu_{u}} .
$$

Here, $u_{\max }, E_{\mathrm{H}}$ are the maximum absolute displacement and dissipated hysteretic energy (excluding elastic energy) under the earthquake (Park \& Ang 1985; Park et al 1985, 1987; Abbas 2006). $\mu_{u}$ is the ultimate yield ductility capacity under monotonic loading and $\beta$ is a 

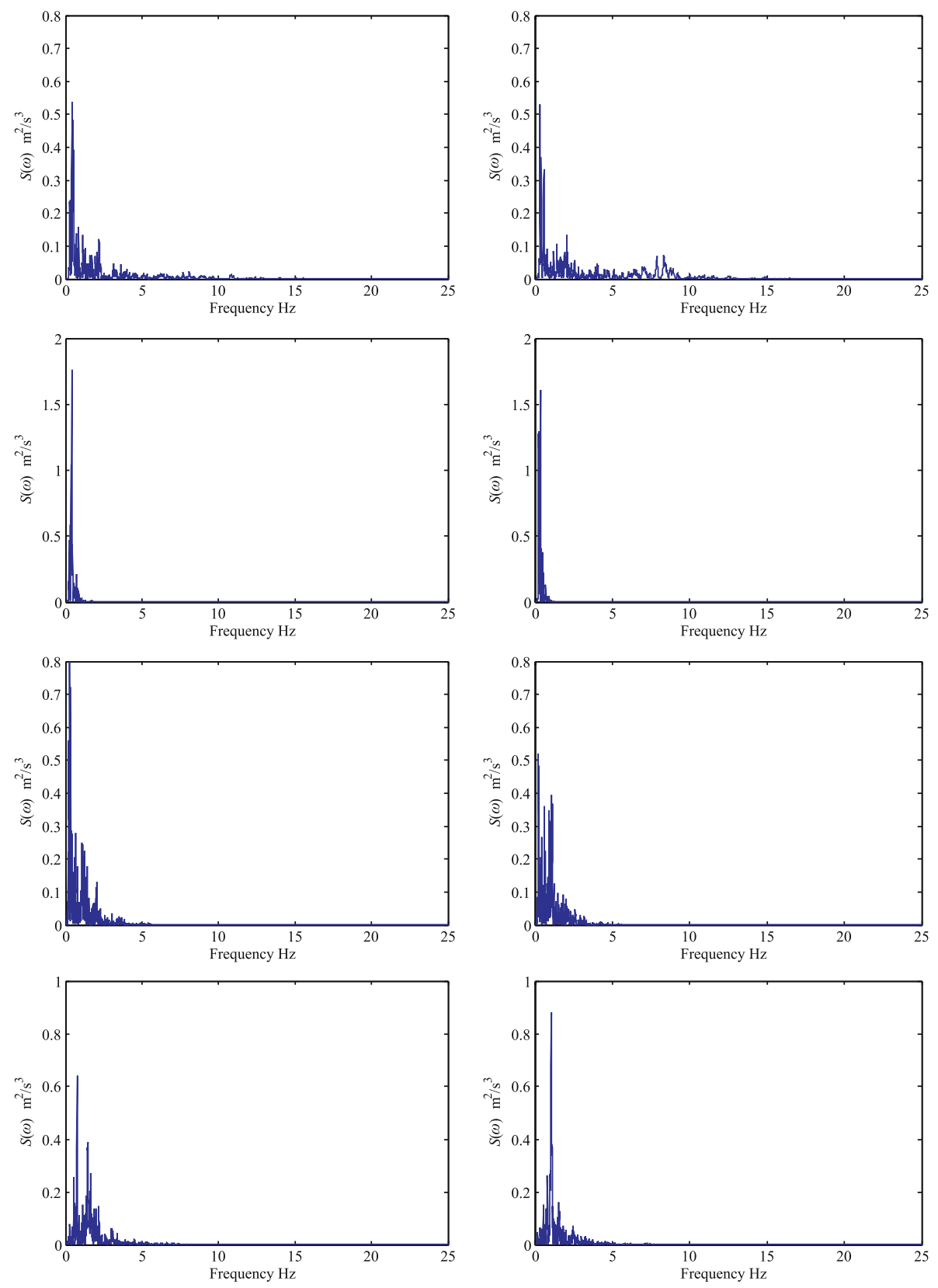

Figure 7. Power spectral density function: Long-duration records.

positive constant that weights the effect of cyclic loading on structural damage (taken as 6 and $0 \cdot 15$, respectively). The numerical results on $D I_{\mathrm{PA}}$ are provided in table 4 . It is evident from these numerical results that the Cape Mendocino record, which has lower entropy and dispersion measures, produces more damage than the Landers acceleration. In fact, the three structures are damaged beyond repair $\left(D I_{\mathrm{PA}}>0.40\right)$ by the first acceleration while they sustain the second acceleration with repairable damage $\left(D I_{\mathrm{PA}}<0.40\right)$. 
Table 4. Ductility factor and damage index for SDOF in elastic structures subjected to ground acceleration.

\begin{tabular}{|c|c|c|c|c|c|c|}
\hline \multirow[b]{3}{*}{ Earthquake (station) } & \multicolumn{6}{|c|}{ Structure fundamental frequency } \\
\hline & \multicolumn{2}{|c|}{$f=0.13 \mathrm{~Hz}$} & \multicolumn{2}{|c|}{$f=0.21 \mathrm{~Hz}$} & \multicolumn{2}{|c|}{$f=0.41 \mathrm{~Hz}$} \\
\hline & $\begin{array}{l}\text { Ductility } \\
\text { factor }\end{array}$ & $\begin{array}{c}\text { Damage } \\
\text { index }\end{array}$ & $\begin{array}{l}\text { Ductility } \\
\text { factor }\end{array}$ & $\begin{array}{l}\text { Damage } \\
\text { index }\end{array}$ & $\begin{array}{l}\text { Ductility } \\
\text { factor }\end{array}$ & $\begin{array}{c}\text { Damage } \\
\text { index }\end{array}$ \\
\hline 1992 Cape Mendocino (CM) & $4 \cdot 39$ & 0.74 & 7.09 & 1.05 & 1.41 & $0 \cdot 19$ \\
\hline 1992 Landers (Lurcene) & 0.93 & $0 \cdot 12$ & $0 \cdot 60$ & 0.08 & 0.43 & 0.05 \\
\hline
\end{tabular}

\section{Concluding remarks}

A measure of the uncertainty or the amount of disorder in earthquake ground motions using the entropy principle is developed in this paper. The uncertainty quantification of the ground motions is important in reliability analysis and performance and optimal design of structures. The entropy rate provides a useful measure of the frequency content of the ground acceleration. Resonant ground motions are shown to possess small entropy while ground motions that are rich in frequency content possess high entropy. An additional measure of the dispersion in the power spectral density function developed by Vanmarcke $(1972 ; 1976)$ is also proposed for characterizing the frequency bandwidth of the ground motion. Bounds on the dispersion and entropy indices of probabilistic and deterministic earthquake accelerations are also estimated. These measures are used for identifying resonant acceleration records. For the limited number of earthquake records considered, the numerical results reveal that the entropy rate and the dispersion indices successfully identify resonant accelerograms.

\section{References}

Abbas A M, Manohar C S 2002 Investigations into critical earthquake load models within deterministic and probabilistic frameworks. Earthq. Eng. Struct. Dyn. 31: 813-832

Abbas A M 2006 Critical seismic load inputs for simple inelastic structures. J. Sound Vib. 296: 949-967

Abbas A M, Manohar C S 2007 Reliability-based vector nonstationary random critical earthquake excitations for parametrically excited systems. Structural Safety 29: 32-48

Abrahamson N, Ashfold S, Elgmal A, Karmer S, Seible F, Somerville P 1998 Proc. First PEER Workshop on characterization of special source effects

Amiri G G, Dana F M 2005 Introduction to the most suitable parameter for selection of critical earthquakes. Comp. Struct. 83: 613-626

Anderson J C, Bertero V V 1987 Uncertainties in establishing design earthquakes. J. Struct. Eng. ASCE 113(8): 1709-1724

Arias A 1970 A measure of earthquake intensity. Seismic design of nuclear power plants, Cambridge, MA: MIT press 438: 468

Bommer J J, Scherbaum F 2005 Capturing and limiting ground-motion uncertainty in seismic hazard assessment. NATO. SCI. S. SS. IV. EAR. 58: 25-40

Bozorgnia Y, Campbell K W 2004 Engineering characterization of ground motion. Earthquake Engineering. Bozorgnia and Bertero (editors), CRC Press, Florida

Cao H, Friswell M I 2009 The effect of energy concentration of earthquake ground motion on the nonlinear response of RC structures. Soc. Dyn. Earthq. Eng. 29: 292-299 
Der Kiureghian A 1996 A coherency model for spatial varying ground motions. Earth Eng. Stuct. Dyn. 25: 99-111

Geller R J, Jackson D D, Kagan Y Y, Mulargia F 1997 Earthquakes cannot be predicted. Science 275(5306): 1616-1620

Housner G W, Hudson D E 1958 The Port Hueneme earthquake of March 18, 1957. Bull. Seism. Soc. Amer. 48: 163-168

Iyengar R N 1989 Critical seismic excitation for structures. Fifth ICOSSAR conference, San Francisco

Iyengar R N, Manohar C S 1987 Nonstationary random critical excitations. J. Eng. Mech. 133: $529-541$

Kalkan E, Kunnath SK 2008 Relevance of absolute and relative energy content in seismic evaluation of structures. Adv. Struct. Eng. 11(1): 1-18

Kanai K 1957 Semi-empirical formula for the seismic characteristics of the ground. Tokyo. Bul. Earthq. Res. Ins. 35: 309-325

Kapur J N 1993 Maximum entropy models in science and engineering. Wiley Eastern, New Delhi

Lee W H K, Shin T C, Kuo K W, Chen K C, Wu CF 2001 Data files from CWB free field strong-motion data from the 21 September Chi-Chi Taiwan earthquake. Bull. Seism. Soc. Amer. 91(5): 1390

Lin Y K 1967 Probabilistic theory of structural dynamics (NY: McGraw-Hill)

Makris N, Black C J 2004 Evaluation of peak ground velocity as a good intensity measure for nearsource ground motions. J. Eng. Mech. 130(9): 1032-1044

Manohar C S, Sarkar A 1995 Critical earthquake input power spectral density function models for engineering structures. Earthq. Eng. Struct. Dyn. 24: 1549-1566

Moustafa A 2009 Discussion of the effect of energy concentration of earthquake ground motion on the nonlinear response of RC structures. Soil Dyn. Earthq. Eng. 29: 1181-1183

Moustafa A 2010 Discussion of analytical model of ground motion pulses for the design and assessment of seismic protective systems. J. Struct. Eng. ASCE, 136: 229-230

Moustafa A, Takewaki I, Wijeyewickrema A 2010 Selection of earthquake records for time-history analysis of structures. Proceedings of the Fifth Conference on Earthquake Engineering, 3-5 March, Tokyo Institute of Technology, Tokyo 1435-1444

Papoulis A, Pillai S U 2002 Probability, random variables and stochastic processes, 4th edition (NY: McGraw-Hill)

Park Y J, Ang A H-S 1985 Mechanistic seismic model for reinforced concrete. J. Struct. Eng. 111(4): 722-739

Park Y J, Ang A H-S, Wen Y K 1985 Seismic damage analysis of reinforced concrete buildings. J. Struct. Eng. 111(4): 740-757

Park Y J, Ang A H-S, Wen Y K 1987 Damage-limiting aseismic design of buildings. Earthquake Spec. 3(1): 1-26

PEER 2005 Pacific Earthquake Engineering Research Center. http://peer.berkeley.edu

Sasani M, Bertero V V 2000 Importance of severe pulse-like ground motions in performance-based engineering: Historical and critical review. Proceedings of 12th WCEE

Seekins L C, Brady A G, Carpenter C, Brown N 1992 Digitized strong-motion accelerograms of North and Central American Earthquakes 1933-1986. CD-ROM, U.S. Geological Survey Digital Data Series (DDS) \#7

Shannon C 1948 A mathematical theory of communication. Bell. Sys. Tec. J. 27

Sigbjornsson R, Ambraseys N 2004 Uncertainty of strong-motion and seismic hazard. Bulletin Earthq. Eng. 1: 321-347

Tajimi H 1960 A statistical method of determining the maximum response of a building structure during earthquakes. Proc. 2nd WCEE, Tokyo 2: 781: 797

Takewaki I 2001 Resonance and criticality measure of ground motions via probabilistic critical excitation method. So. Dyn. Earthq. Eng. 21: 645-659

Vanmarcke E H 1972 Properties of spectral moments with applications to random processes. J. Eng. Mech. 42: 215-220 
Vanmarcke E H 1976 Structural response to earthquakes. Seismic risk and engineering decisions. Lomnitz C, Rosenbluth E (editors) (NY: Elsevier)

Wen Y K, Ellingwood B R, Veneziano D, Bracci J 2003 Uncertainty modelling in earthquake engineering. MAE Center Project FD-2 report

Zhai C-H, Xie L-H 2007 A new approach of selecting real input ground motions for seismic design: The most unfavourable real seismic design ground motions. Earthquake Eng. Struct. Dyn. 36: $1009-1027$ 